\title{
THE GENESIS OF THE NORMAL ELECTROCARDIOGRAM
}

\author{
BY \\ ATHELSTANE HILL \\ From Newton Abbot Hospital, South Devon \\ Received March 28, 1946
}

The argument of this paper is based on the great work of Lewis, and all references to "Lewis, 1925" are to the third edition of The Mechanism and Graphic Registration of the Heart Beat. His demonstration (confirming Engelmann, 1875) that the contraction wave passes through cardiac muscle from the point of stimulation in all directions with equal velocity (p. 84); his considered view of the direction of the spread of the excitation wave in the human ventricles (diagram, p. 115); his table of synchronous events (p. 47); and his statement that the direction of travel of the contraction wave is of more importance than the disposition of contracting and resting masses of muscle in determining the form of the electrocardiogram (p. 57, footnote) are of fundamental importance.

In this paper two other points are emphasized : that air-filled lung is practically an insulator; and that the laws of composition and resolution of mechanical forces do not apply to electrical currents. On these concepts an explanation differing in some respects from that given by Lewis is founded.

\section{The Fundamental Principle}

If a battery, packed in insulating material, is placed inside a hollow shell of conducting material, with its positive and negative poles connected, through resistances $p$ and $n$, with areas $\mathrm{P}$ and $\mathrm{N}$ on the inner surface of the shell, a current will flow from $\mathrm{P}$ to $\mathrm{N}$ through the wall of the shell in all directions (Fig. 1). If the terminals of a voltmeter are applied to any two points, A and B, on the surface of the shell, the voltmeter will register the difference of potential between them.

The heart is a battery : it is packed in insulating material, air-filled lung; its positive and negative plates are the anterior and posterior faces of an advancing contraction wave ; and $p$ and $n$ represent the resistances of the conducting tissues leading from these "plates" to the wall of the thorax. The thorax is a hollow, hemi-ellipsoidal shell whose walls are made of conducting materials, viz. blood-filled muscles and, through the diaphragm, the liver. $\mathrm{P}$ and $\mathrm{N}$ are the most positive and negative points on the surface-the surface poles-and the string galvanometer is for all practical purposes a voltmeter, as it is standardized before each lead to register volts, not amperes.

\section{INTRINSIC AND SURFACE VolTAGE}

The difference of potential between two points on an electrical circuit is proportional to the resistance between those points. The total resistance of the circuit from the + plate to the - plate is $p+s+n$, where $s$ is the resistance of the shell. If $\mathrm{V}$ is the potential difference between the plates - the intrinsic voltage of the battery-then the potential difference, $\mathrm{E}$, between the surface poles $\mathrm{P}$ and $\mathrm{N}$-the surface voltage-will be $\mathrm{V}\left(\frac{s}{p+s+n}\right)$. If $s$ is constant the relation of $\mathrm{E}$ to $\mathrm{V}$ depends on the values of $p$ and $n$; if either is large, $\mathrm{E}$ will be small. But if these are constant and $s$ variable, a decrease in $s$ will diminish the ratio $\frac{E}{V}$ 
$+{ }^{+} \quad$ Positive and negative plates of battery.

$\begin{array}{ll}p, n \text {. } & \text { Positive and negative " surface poles." } \\ \text {. } & \text { Pesistances between plates and poles. }\end{array}$

A, B. Points on the surface of the shell, connected by a voltmeter (not shown in diagram).

$r_{1}$. Resistance between $\mathbf{P}$ and $A$ on circuit $\mathbf{P}-\mathbf{A}-\mathbf{N}$.

$r_{2}$. Resistance between $\mathrm{P}$ and $\mathrm{B}$ on circuit $\mathrm{P}-\mathrm{B}-\mathrm{N}$.

It can be shown mathematically that the resistance of a hollow metal sphere of uniform thickness and conductivity is practically constant wherever the poles are applied, unless they are very close together. If Fig. 1 represents a sphere of 36 in. circumference, and $\mathbf{P}$ and $\mathbf{N}$ are circular areas 2 in. in diameter, the resistance $s$ will vary by less than \pm 10 per cent from a mean value so long as the centres of $P$ and $N$ are not less than 4.5 in. apart. But at 3 in. the resistance is halved, and at 2 in. it is zero; for then the edges of $\mathbf{P}$ and $\mathrm{N}$ are in contact and the current is short-circuited without passing through the shell.

It will be seen that low surface voltage, without change of intrinsic voltage, may be due to poor contact between the heart and the chest-wall at one or both of the poles, to proximity of the surface poles, and to internal short-circuiting. These account for the low voltage of some phases of the normal cycle.

In pathological conditions low surface voltage (i.e. low voltage in all leads, including the most favourable chest lead) is sometimes thought to be evidence of degeneration of the myocardium or of interference with its function, e.g. by tamponnade. This is not necessarily the case. In advanced emphysema the interposition of a pad of lung between the heart and the chest wall may cause very low surface voltage for many years before there is any sign of heart failure. Pericardial effusion may have the same effect. It is possible that the low voltage associated with manifest heart failure may sometimes be due to the presence of fluid in the pericardium.

It will also be seen that the "surface zero"- the potential intermediate between $\mathbf{P}$ and $\mathbf{N}$ -bears no fixed relation to the " intrinsic zero." If $n>(s+p)$ the whole surface of the shell, including $\mathrm{N}$ itself, must be positive. No point or combination of points can be found on 
the body that will give a true zero potential, or will be at the same potential throughout the cycle, or will be at the same potential at the same phase of the cycle in different patients. The various forms of two-point and three-point lead may prove clinically useful, but they do not give absolute voltage values, comparable from patient to patient, and it would surely be advisable to avoid terms that suggest that they do, such as unipolar lead, voltage lead, indifferent point, etc.

\section{Potential distribution in the Normal Cycle}

Fig. 2 to 6 and 8 show the main features of the potential distribution on the chest, with diagrams of the corresponding events in the heart. In constructing the diagrams of ventricular excitation the time-relations given by Lewis (1925) have been followed. The contraction wave is shown in the diagrams as a line between shaded and unshaded muscle. The shaded area is in contraction, and is conducting negative electricity from the posterior face of the wave; the unshaded part is about to pass into contraction, and is conducting positive electricity from the anterior face of the wave; arrows show the paths of conduction. In the surface charts the shaded areas are positive, the stippled areas negative; the poles are shown as clear spaces with $a+$ or - sign.

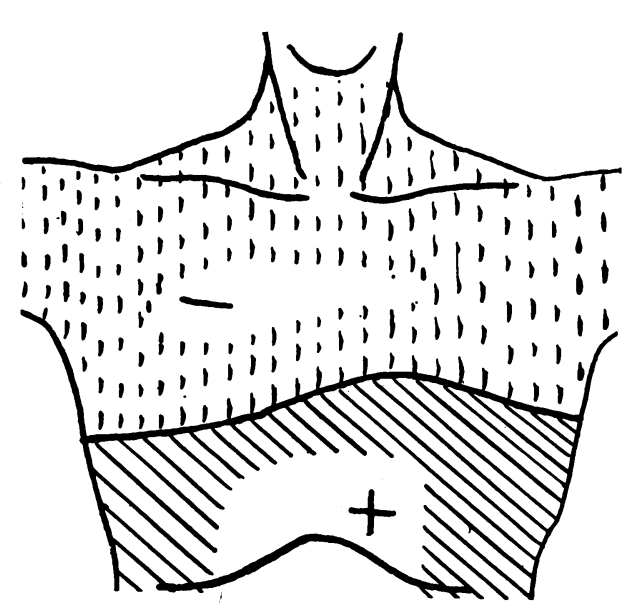

A

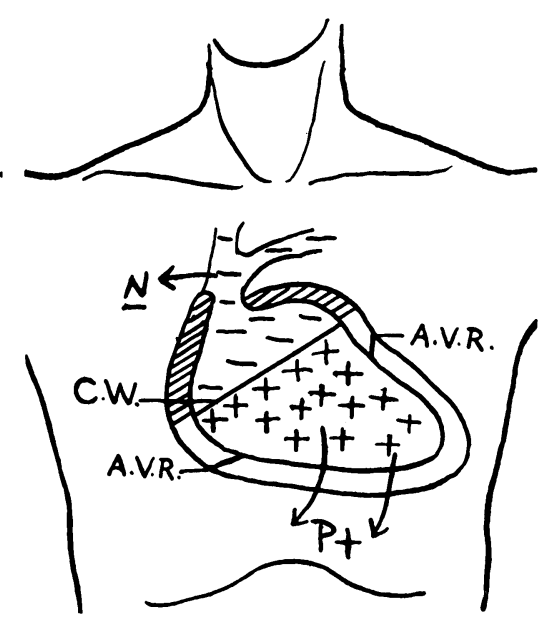

B

FIG. 2.-The $P$ wave.

(A) Potential distribution on chest.

(B) Electrical events in the heart. A.V.R., auricular-ventricular ring. C.W., contraction wave. P, N, surface poles.

In Fig. 2B the wave has passed half-way down the auricles; these have begun to contract, and a wave of distension is starting to travel backwards from the superior vena cava. This distension wave reaches its maximum in the jugular veins during the inscription of QRS (Lewis, 1925)-the $a$ wave of the polygram. It forms the negative pole. At the present stage its contact with the chest wall is poor-resistance $n$ is large-and the surface voltage is low. There is also free internal short-circuiting through the intra-auricular blood.

In Fig. 3 the stimulus reaches both sides of the septum simultaneously. Both surfaces become negative, and there is no conduction path for the positive electricity in the interior to the chest wall except down the septum to the apex, where the positive pole is in immediate contact with a negative area that surrounds it. Shell resistance, $s$, is therefore minimal, and the surface voltage so low that there is no deflection.

The stimulus next reaches the papillary muscles (Fig. 4). There is not much shortcircuiting, but again the poles are close to one another and the surface voltage is low.

The stimulus now strikes the endocardial surface of both apices and travels up both arborizations (Fig. 5 and 6). If the stimulus passes along the arborisation with velocity $a$, and at every point as it passes starts a contraction wave which spreads radially with velocity 


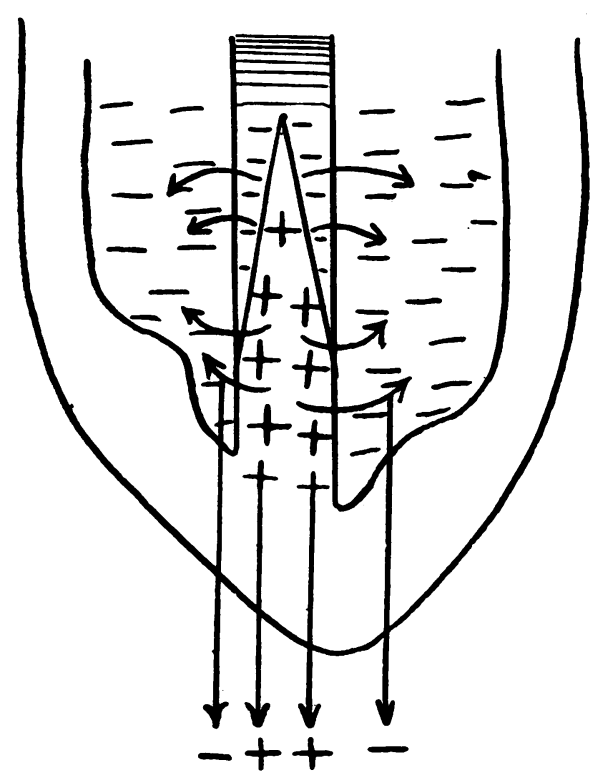

FIG. 3.-Stimulation of ventricular septum.

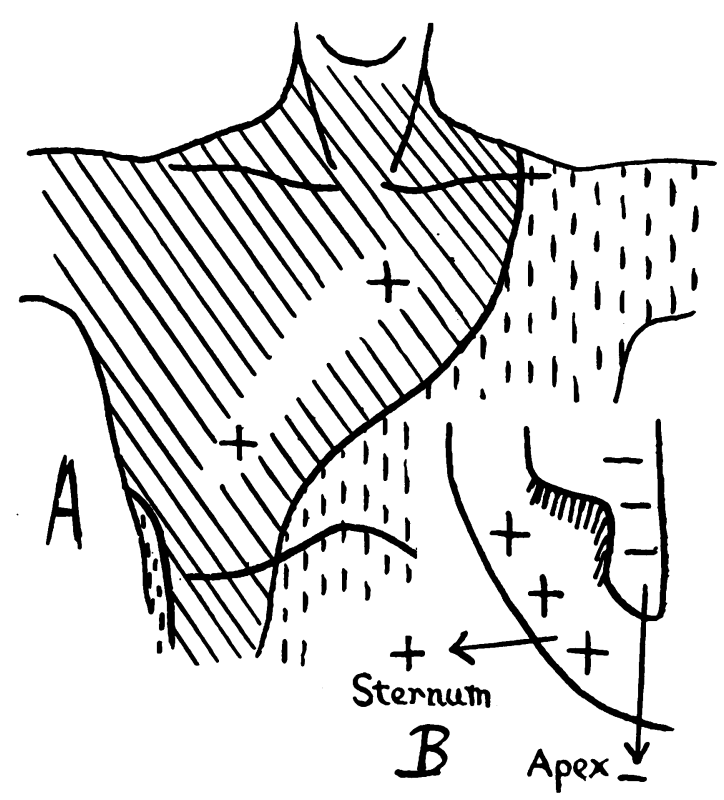

Fig. 4.-Stimulation of papillary muscles.

(A) Potential distribution during inscription of $Q$. (B) (inset) Corresponding electrical events. The negative pole (not shown because of the inset) is at the apex.

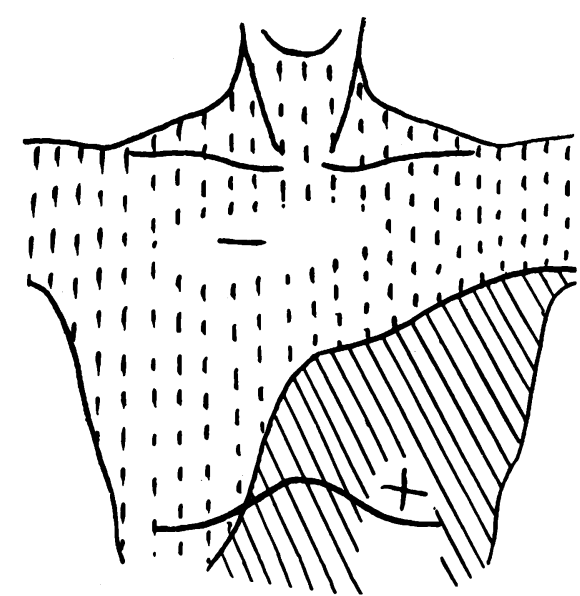

A

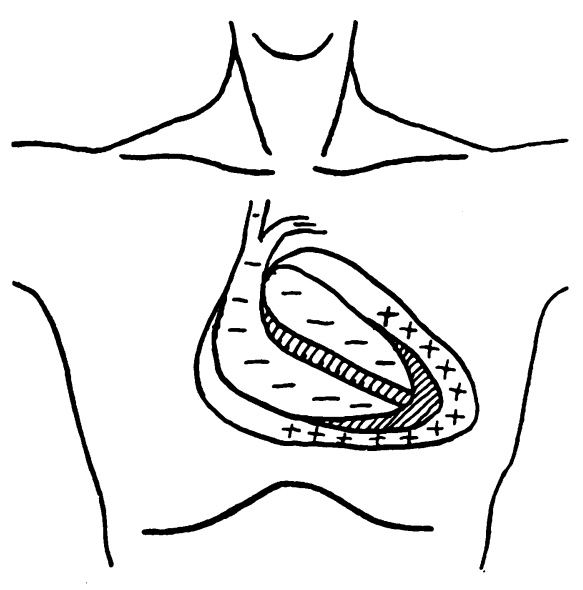

B

FIG. 5.-The first part of the RS deflection.

(A) Potential distribution. (B) Electrical events.

$c$, the net result is a continuous contraction wave travelling away from the endocardium at an angle whose tangent is $a / c$. Meanwhile the distension wave has reached the innominate veins and is spreading across behind the manubrium from right to left. Both poles are now in close contact with the chest wall, and are not close together. $p$ and $n$ are small, $s$ is large; the ratio $\mathrm{E} / \mathrm{V}$ is at its largest and the surface voltage is high.

The left ventricle is thicker than the right, and the contraction wave takes a little longer to pass right through its wall. The effects of this are shown in Fig. 6.

On this view, septal contraction takes place during the last part of the P-R interval, the phase of silent activity. Comparison of normal and abnormal complexes in the same lead from the same patient shows that in the "short P-R, B.B.Bl. syndrome" (Hunter, Papp, and Parkinson, 1940) the part of the QRS deflection that precedes the notch does actually occupy this period. The features of this syndrome may be explained by supposing that there is a 

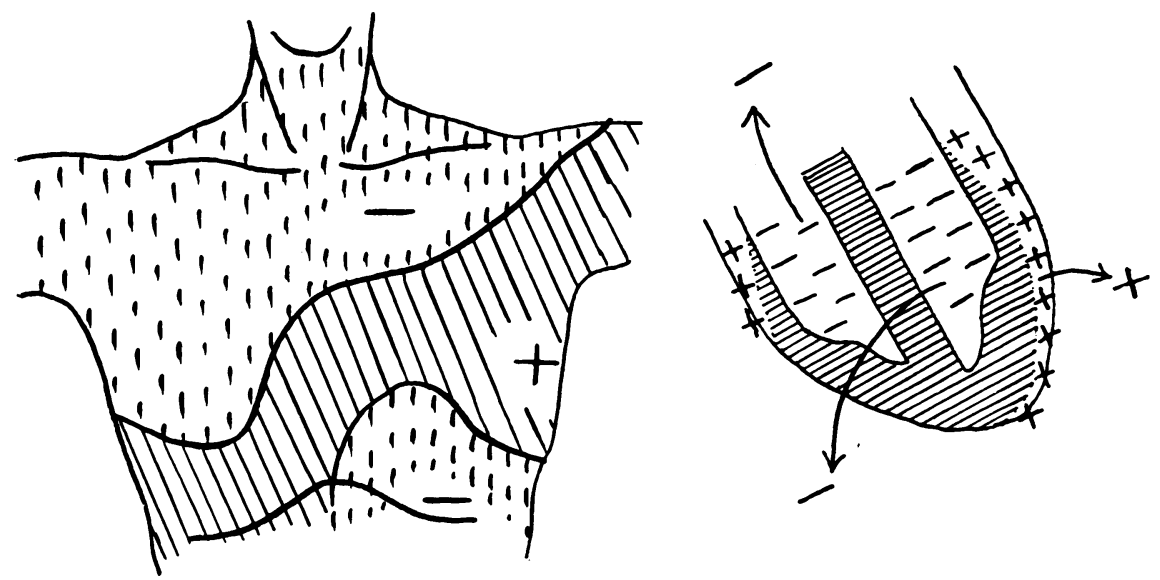

FIG. 6.-End of QRS deflection. Potential distribution and electrical events at the end of R I.

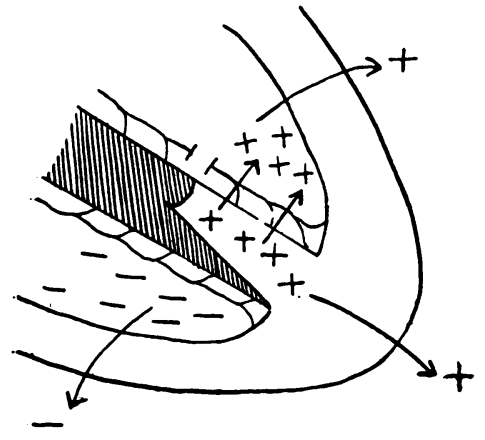

A

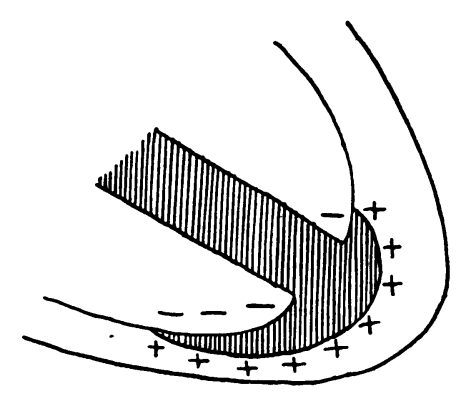

B

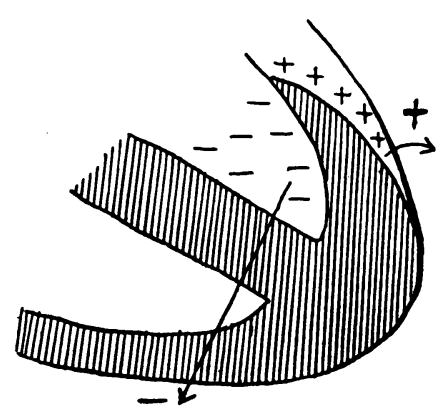

C

FIG. 7.-Sequence of electrical events during inscription of QRS in organic left bundle branch block.

(A) The initial deflection. (B) The "notch." (C) End of the deflection.

shorter or longer delay in the passage of the stimulus down the left bundle branch. The first deflection is then due to unilateral stimulation of the septum; the form of the rest of the complex depends on the degree of asynchronism. Fig. 7 shows the sequence of electrical events in organic left bundle branch block; it is easy to see that these would give the typical electrocardiogram. This explanation has been suggested elsewhere (Hill, 1939) and independently by Roche (1945).

\section{THE T WAVE}

The only mystery of the $T$ wave is its direction. When a strip of cold-blooded muscle is stimulated at one end the contraction wave deflection is followed after an interval by an anabolic wave deflection which is flatter, broader, and opposite in direction. The $\mathrm{T}$ wave follows the ventricular contraction wave after an interval, and is broader and flatter, but not necessarily in the opposite direction. There can be little doubt that it is anabolic; it is modified by many conditions that are known to affect anabolism-asphyxiation, ischæmia, diabetes, etc. Moreover, a ventricular extrasystole can occur immediately after it, or even during its downstroke, but never before it. This proves that some degree of anabolism occurs during its inscription.

But there is one form of $T$ wave that does follow the pattern of the muscle strip-the $T$ wave of a ventricular extrasystole; it is broader, flatter, and always in the opposite direction to the primary deflection. That it is an anabolic wave is proved by the fact that another ventricular extrasystole can follow immediately after it.

In the auricles mechanical contraction, i.e. diminution of cubic capacity with expulsion of contents, follows almost immediately on the electro-chemical event that causes the $\mathbf{P}$ wave; in the ventricles mechanical contraction begins during the $\mathrm{S}-\mathrm{T}$ interval. Lewis' table of 
synchronous events shows that the inscription of QRS is completed before the aortic valves open; QRS is synchronous with a sharp rise of intra-ventricular pressure to the level of the aortic pressure. During, and for some time after, this period the ventricular walls are put on the stretch, and the blood is squeezed out of them (systolic blanching can easily be seen in the exposed frog's heart). Shortly after the opening of the aortic valves the pressure in the aorta rises rather higher than in the ventricle; it is at this point that the $T$ wave begins, and its peak coincides with the maximum difference of pressure at this stage. It comes to an end shortly before or after the aortic valves close ; the $U$ wave accompanies the dicrotic rise in the aorta.

The distinctive feature of a ventricular extrasystole is that it causes little rise of intraventricular pressure; the walls do not become ischæmic, and the anabolic wave is able to follow its natural course in the wake of the contraction wave. With a normal contraction, anabolism is prevented by ischæmia; it has to wait for the return of blood, which penetrates inwards from the coronary vessels on the surface. It therefore travels in the opposite direction to the contraction wave, and, being of opposite sign, gives a similar deflection. It travels relatively slowly compared with the contraction wave, and most of the time the whole of both ventricles is taking part in it. Distension of the pulmonary artery and re-expansion of the right auricle give a wide distribution of negative potential, different from that obtaining during inscription of QRS, so that the T wave is not an exact copy of it in every lead (Fig. 8).

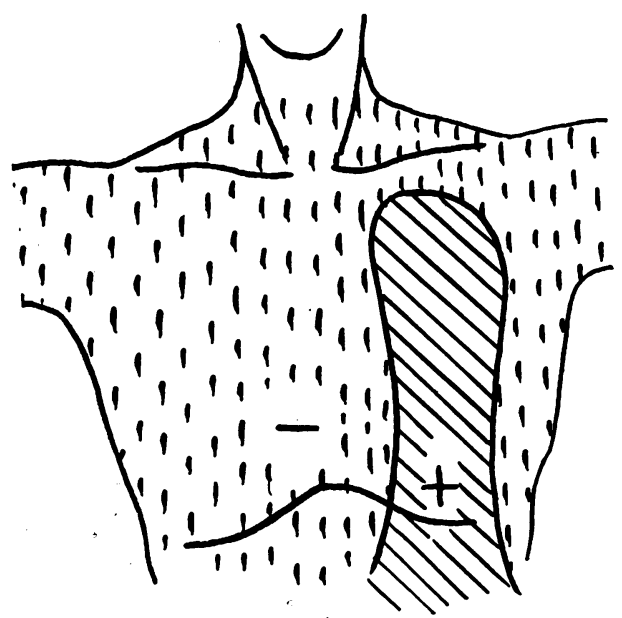

A

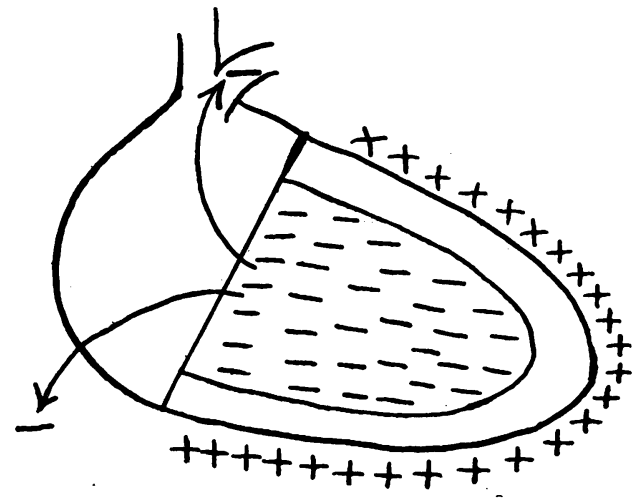

B

Fig. 8.-Peak of $\mathrm{T}$ wave. (A) Potential distribution. (B) Electrical events.

In short, the explanation offered is that the return wave of a ventricular extrasystole is the "natural" anabolic wave, and the normal $\mathrm{T}$ wave is the same thing modified by intraventricular pressure. When the aortic pressure during diastole is high and the left ventricle is beginning to fail its wall may be so much stretched that the coronary return is delayed till relaxation is far advanced; the $T$ wave is then diphasic. Reduction of peripheral pressure by trinitrin reduces the intraventricular tension, and the $T$ wave may then become upright and normal.

Delay of anabolism of the left auricle by intra-auricular tension may perhaps account for the onset of flutter or fibrillation in mitral stenosis.

\section{INSCRIPTION OF DEFLECTIONS}

In the normal subject there is a point at the costal margin, about three inches to the left of the middle line of the back, that is iso-electric with the standard left leg contact throughout the cycle. The left leg contact is virtually attached to the thorax at this point; similarly the forearm contacts are virtually attached to the thorax high up in the axillæ, the arms functioning simply as additional lengths of wire of low resistance.

It is convenient to think of the conduction paths between the surface poles, $P$ and $N$, as 
an infinite number of separate circuits lying side by side. Fall of potential between two points on a circuit is proportional to the resistance between those points. In Fig. 1, if $\mathrm{E}$ is the potential difference between $\mathrm{P}$ and $\mathrm{N}, \mathrm{R}_{1}$ the resistance of the circuit $\mathrm{P}-\mathrm{A}-\mathrm{N}$, and $r_{1}$ the resistance of that part of it lying between $P$ and $A$, the fall of potential from $P$ to $A$ is $E\left(r_{1} / R_{1}\right)$. If, by a similar notation, the fall of potential from $P$ to $B$ is $E\left(r_{2} / R_{2}\right)$, the potential difference between $A$ and $B$, registered by the voltmeter, will be $E\left(r_{2} / R_{2}-r_{1} / R_{1}\right)$.

The positions of the surface poles during inscription of $R$ in lead 1 are shown in Fig. 5A. The negative pole moves leftward to the position shown in Fig. 6, following the backward movement of the distension wave along the innominate vein. In Fig. 9 the left axilla corresponds with A in Fig. 1, the right axilla with B. Since the conducting musculature is much the same on both circuits it is clear that $r_{2} / R_{2}$ is at first much greater than $r_{1} / R_{1}$; conse-

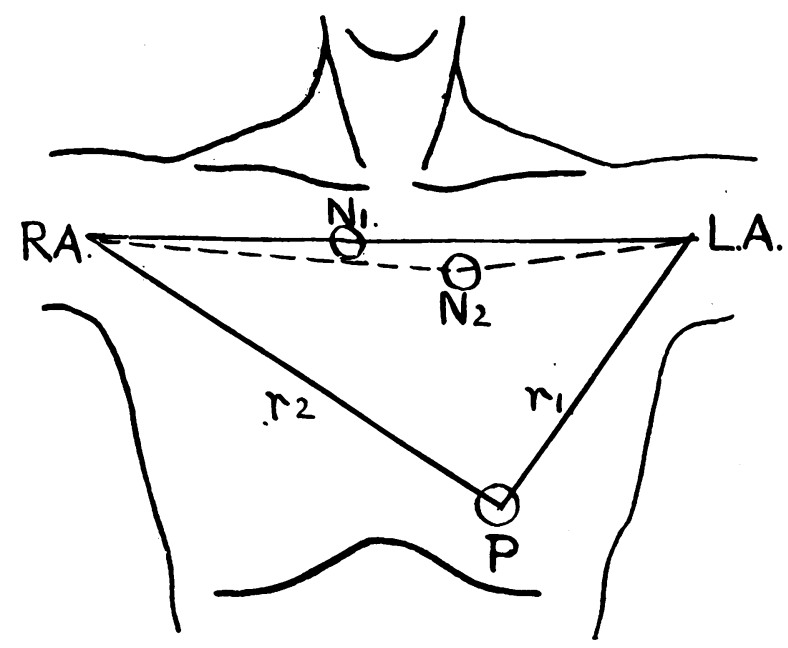

FIG. 9.-Diagram illustrating inscription of $\mathbf{R} \mathbf{I}$.

quently L.A. will be strongly positive to R.A., and there will be a large upward deflection. But as $\mathrm{N}$ moves leftward from $\mathrm{N}_{1}$ to $\mathrm{N}_{2}, \mathrm{R}_{2}$ increases at the expense of $\mathrm{R}_{1}$ while $r_{1}$ and $r_{2}$ remain unchanged. The difference between L.A. and R.A. therefore diminishes and the deflection falls back to the base line. A little further movement of $\mathrm{N}$ to the left would invert it.

Second and third lead deflections are determined by the same principles, but as the conduction paths from the left leg point pass through the dorsal muscles their amplitude and direction vary with the shape and muscular development of the chest.

\section{EleCtrical AXIS}

It is not too much to say that the whole doctrine of electrical axes, resultant and component currents, right and left preponderance, summation of currents, equilateral triangles, etc. is quite untenable and must be abandoned. The direction of an electrical current is determined solely by the conducting paths available and has no relation to the orientation of the battery in space. Electrical currents do not "sum" unless their sources are connected in series-the negative pole of one to the positive pole of the other and vice versa. When connected in parallel the voltage is intermediate between that of the two sources, the exact value depending on the arrangement of resistances, external and internal. Simultaneous occurrence of an auricular contraction and a ventricular event is equivalent to connection in series (combine Fig. 2B with Fig. 5B or 8B); the $P$ wave appears superimposed unchanged on the QRS or T. But the two ventricles are connected in parallel, having a negative contact in common and a continuous positive pole (Fig. 5B and 8B); it is impossible that their voltages should be added.

It is true that if the ends of a diameter of a mass of conducting material are maintained at a potential difference $\mathrm{V}$, a voltmeter applied to the ends of other diameters will show intermediate voltages resembling the components of a mechanical force; but the law determining 
their magnitude would depend entirely on the shape of the conducting mass. A component of a mechanical force is proportional to the cosine of the angle between its direction and the line of the force, but this would be true of electrical components only if the mass were a straight rod of uniform cross-section. If the mass were a sphere the voltmeter would show a voltage equal to $\mathrm{kV} \cdot \log \left(\frac{1+\cos \mathrm{A}}{1-\cos \mathrm{A}}\right)$; with other shapes other rules would hold good.

But when we recognize that air-filled lung is almost an insulator the picture becomes that shown in Fig. 1. Here the orientation of the battery matters not at all; all that matter are the points at which the paths of least resistance from the plates of the battery most nearly approach the inner surface of the conducting shell. These become the surface poles, and potential distribution then follows the ordinary laws of conduction through the walls of the shell.

It is, then, difficult to assign any precise meaning to the term electrical axis; but right and left axis deviation are convenient labels for two contrasted types of electrocardiogram.

Left axis deviation. The tall R I and deep S III indicate that at the peak of the QRS deflection the left axilla is strongly positive to the other two contacts; (similarly, the deep S I and tall R III of R.A.D. show that the left axilla is strongly the most negative of the three). Left axis deviation is common with enlargement of the ventricles, but has never been satisfactorily correlated with preponderance of the left ventricle (Lewis, 1928). All that is necessary for its appearance is that the positive pole, formed by the apical halves of the ventricles, shall be electrically closer than usual to the left axilla. The mere anatomical displacement of the apex is enough to secure this, but when the heart is much enlarged collapse of the base of the left lung must contribute to the effect.

The natural electrical action of the heart was imitated in a series of experiments by applying the poles of a battery (with suitable precautions) to various points on the chest of a subject attached to an electrocardiograph and noting the deflections resulting.

In the first experiment the negative pole was fixed to the middle of the sternum at the level of the second space. Applying the positive pole to the apex beat gave a large upward deflection in lead I and also in lead III; their relative amplitudes were comparable to those of R I and R III in the subject's standard cardiogram. Moving the positive pole to the sixth rib in the anterior axillary line inverted the deflection in lead III, giving the picture of L.A.D. Moving it inward, by centimetre steps, horizontally from the apex to the middle line gave smaller and smaller upward deflections in lead I, but no S I appeared till the middle line was crossed.

Fig. $10 \mathrm{~A}$, from a patient whose heart was seen by X-rays to be absolutely vertical and centrally placed, shows that this procedure is a fair imitation of the natural event. The first lead deflections are tiny, but not inverted.

A positive pole at the sixth rib in the anterior axillary line will give the picture of L.A.D.; a natural positive pole is in this position when the ventricles are enlarged; is any more elaborate explanation necessary?

Right axis deviation. When this is present the right ventricle is nearly always hypertrophied, but the converse is not always true. In the early stages of mitral stenosis and in advanced emphysema there may be right ventricular hypertrophy without a trace of R.A.D.

In the course of the experiment described above the conditions that would obtain in " right preponderance" were imitated in an exaggerated form. The positive pole was applied to points on the chest over the right ventricular contact, the voltage was at least ten times as great as the intrinsic voltage of any heart, and there was no positive pole at the apex to represent the left ventricle; yet no downward deflection could be produced in lead I as long as the negative pole was in the middle line.

In the second experiment conditions were reversed. The positive pole was fixed at the apex-beat and the negative pole moved to the left, centimetre by centimetre, at the level of the second space. The upward deflection in lead III increased rapidly, but in lead I it decreased, and at $4.5 \mathrm{~cm}$. from the middle line it disappeared; $1 \mathrm{~cm}$. further to the left gave a deep S I and a tall R III-the picture of R.A.D. 
The same result was obtained with the positive pole $4 \mathrm{~cm}$. to the left of the apex in the fifth space, the point of no deflection being $5 \mathrm{~cm}$. from the middle line.

It is clear, then, that a negative pole a very little further to the left than the natural negative pole at the end of the normal R I will give a downward deflection in lead I; and there are two structures in this position, conductors of negative electricity at this stage, which, when dilated, come forward to approach the thoracic wall-the left auricular appendage and the pulmonary artery. The role of a dilated pulmonary artery was suggested by Dr. John Parkinson; it brings the R.A.D. of infundibular stenosis into line with this theory (O'Farrell, 1938).

When these structures are dilated the right ventricle is always hypertrophied, but again the converse is not always true. In early mitral stenosis the right ventricle may be enlarged before much dilatation of the left auricle and pulmonary artery is evident; in emphysema the appendage is not dilated, and the artery is covered by a pad of distended lung.

Fig. 10B affords an indirect confirmation of this view. The patient was a man of 31 , with perfectly normal heart, arteries, and blood pressure. In the second left space was a pulsating tumour; the maximum pulsation was $7 \mathrm{~cm}$. from the middle line, dulness to percussion extended $2 \mathrm{~cm}$. further to the left and down into the third space. X-rays showed a solid tumour in contact with the base of the left ventricle and moving with it. The cardiogram shows extreme left axis deviation, but the peaks come rather later than usual, and coincide with the moment when the stimulus reaches the base of the left ventricle. At this instant positive electricity was conducted from the surface of this area through the tumour to the chest wall at the point of pulsation, making the left axilla strongly positive.

The last experiment described above reproduces the conditions present when the heart is displaced bodily to the left by deformity of the chest wall. Fig. 10C is from such a case.

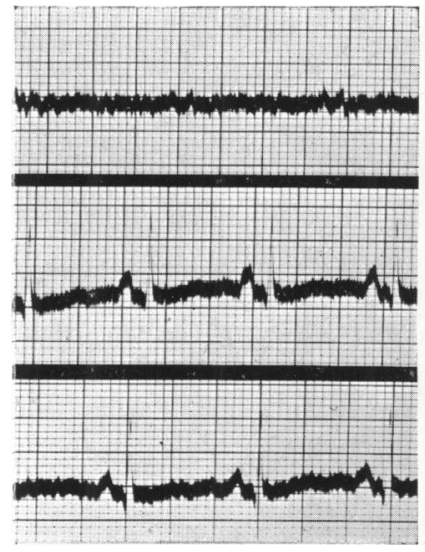

A

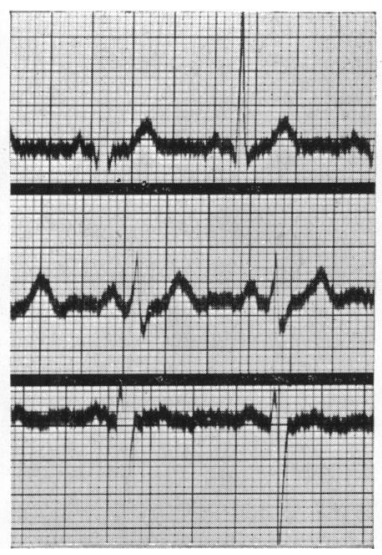

B

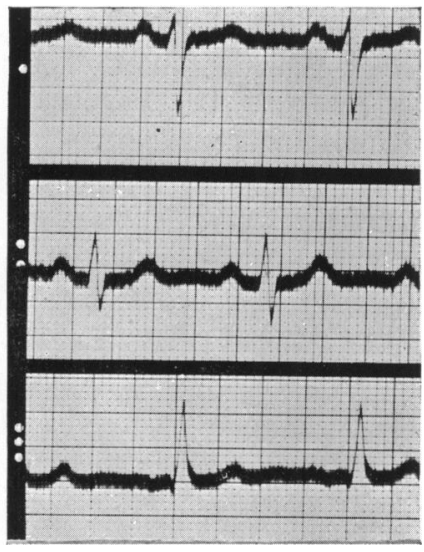

C

Fig. 10.-Electrocardiograms of : (A) Vertical, centrally placed, heart. (B) Left axis deviation due to an intra-thoracic tumour. (C) Right axis deviation due to displacement of the heart by deformity of the chest. (The second complexes in leads I and III of Fig. 10B have been traced over in Indian ink to facilitate reproduction.) (Reduced to two-thirds.)

X-rays showed no clock-wise rotation of the physical axis of the heart and no sign of hypertrophy of the right ventricle, nor was there any clinical reason to suspect this. The cardiogram shows marked R.A.D.

The argument may be summed up as follows.

1. While the negative pole is in the middle line no voltage applied to the left of the middle line can produce a downward deflection in lead $\mathrm{I}$.

2. A negative pole in the second space $6 \mathrm{~cm}$. to the left of the middle line reproduces the picture of R.A.D. even when the positive pole is to the left of the apex-beat.

3. There are structures, negative during QRS, which when dilated approach the chest wall at this point. 
4. When these structures are dilated the right ventricle is always enlarged, but the converse is not always true.

5. When R.A.D. is present the right ventricle is nearly always enlarged, but the converse is not always true.

6. In both cases dissociation occurs in the same circumstances.

7. Exceptionally R.A.D. may be present without rotation of the physical axis of the heart or enlargement of the right ventricle.

8. A pathological positive pole $7 \mathrm{~cm}$. to the left of the middle line in the second space produces extreme L.A.D.

Is it not reasonable to suppose that a pathological negative pole, known to be present in this region, may be the cause of R.A.D.?

Further, is there any direct evidence that the intrinsic voltage of a hypertrophied right ventricle is much greater than that of a normal left ventricle? or indeed of a normal right ventricle? If not, the doctrine of electrical preponderance has no basis whatever.

Salient points have already been discussed in the course of this paper; it only remains to emphasize that anatomical relations and physical principles are of paramount importance in the interpretation of electrocardiograms.

\section{SUMMARY}

Fundamental principle. The heart is compared to a battery packed in insulating material and enclosed in a hollow conducting shell.

Intrinsic and surface voltage. The ratio of the maximum potential difference on the surface of the shell (the surface voltage) to the voltage of the battery (the intrinsic voltage) is determined by resistance relations. The low voltage sometimes found in emphysema and with pericardial effusion is thus explained.

Potential distribution in the normal cycle. Charts of this, with diagrams of the corresponding events in the heart, are given for each deflection.

The $T$ wave. The $\mathrm{T}$ wave of a ventricular extrasystole corresponds to the secondary wave of a stimulated muscle-strip, and the normal $T$ wave is the same thing modified by intraventricular pressure. Reasons are given for believing it to be anabolic.

Inscription of deflections. The principles determining the direction and amplitude of deflections are explained.

Electrical axis. The laws of composition and resolution of mechanical forces do not apply to electrical currents. Left axis deviation is ascribed to proximity of the positive apex to the left axilla; right axis deviation to an abnormal negative pole between the sternum and the left axilla, formed by dilatation (or occasionally displacement) of the left auricular appendage or pulmonary artery or both. These conditions can be imitated by applying the poles of a battery to appropriate areas on the chest.

Thanks are due to Miss Loveday Hill for invaluable help in preparing the illustrations; and to Sister Lawson, S.R.N., for the photographic work.

\section{REFERENCES}

Englemann, T. W. (1875). Arch. ges. Physiol., Bonn, Bd. 11, 465 (quoted in Schafer's Physiology, 1900 edition, Vol. II, p. 443).

Hill, A. (1939). Lancet, 2, 979.

Hunter, A., Papp, C., and Parkinson, J. (1940). Brit. Heart J., 2, 107.

Lewis, T. (1925). Mechanism and Graphic Registration of the Heart Beat, 3rd edition. Shaw and Sons London.

- (1928). Clinical Electrocardiography, Shaw and Sons, London, p. 36.

O'Farrell, P. T. (1938). Irish J. med. Sci., Sixth series, September 1938, p. 597.

Roche, E. H. (1945). Brit. Heart J., 7, 121. 